\title{
Prevention and treatment of the psychological consequences of trauma in children and adolescents
}

\author{
BENEDETTO VITIELLO
}

There is increased awareness that children are commonly exposed to traumatic events with potential to cause psychological sequelae. Worldwide, children account for many of the victims of wars or natural disasters. Even in the more economically developed countries, it can be estimated that about a quarter will have encountered some severe trauma by the time they are 18 year old (Stevenson, 1999; Costello et al., 2002). Exposure to domestic, school and community violence are especially common in the United States. Terrorism has recently added another dimension to the possible sources of trauma. The rapid spread of information through the media means that more people are exposed, though indirectly, to traumatic events and can suffer negative psychological consequences, primarily because these events undermine the sense of security of the individual and lead to anxiety.

Though most traumatized children eventually recover after a period of acute stress, a sizeable portion develop trauma-induced psychopathology, such as the protracted autonomic, cognitive, and behavioral symptoms of post traumatic stress disorder (PTSD), or other disorders of anxiety, depression, and conduct. Moreover, childhood trauma is also a risk factor for developing PTSD as an adult (Breslau, 2002), suggesting that early stress can

Address for correspondence: Dr. B. Vitiello, Child and Adolescent Treatment and Preventive Intervention Research Branch, National Institute of Mental Health, Room 7147, 6001 Executive Blvd., MSC 9633, Bethesda, MD 20892-9633 (USA).

Fax: +1-301-443.4045

E-mail: bvitiell@mail.nih.gov

Declaration of Interest: The opinions and assertions contained in this report are the private views of the author and are not to be construed as official or as reflecting the views of the Department of Health and Human Services or the National Institutes of Health. cause behavioral sensitization to subsequent stressors. In fact, there are indications that developing organisms are especially vulnerable to stress and its long-lasting consequences (Heim et al., 2000). Thus, even if precise estimates of trauma exposure and prevalence of post-traumatic psychopathology among children are currently not available, the development of effective interventions to prevent and treat PTSD in children has become a prominent public health priority (Friedman et al., 2003). But, how close are we to achieving these goals and what are the challenges that researchers in this field have to contend with?

\section{PREVENTION OF POST-TRAUMATIC PSYCHOPATHOLOGY}

Since the early observations on combat-exposed war veterans more than fifty years ago, substantial progress has been made toward elucidating the pathogenesis and accompanying features of PTSD. It is now recognized that a variety of traumas can lead to PTSD, such as being the victim of a life-threatening event, or of an event that threatens one's physical integrity, or witnessing such an event, or even learning of such an event occurring to a family member or a personally important person (American Psychiatric Association, 1994). The possible source of PTSD has thus become more heterogeneous.

When an individual experiences a trauma, acute neurophysiological and psychological reactions usually occur, whose nature and intensity are influenced by type of trauma, perceived level of threat, trauma context, and pretraumatic conditions. The effects of trauma on a child are influenced by the genetic substrate of the individual and a host of environmental factors, such as social context, parental reactions, family cohesion, and previous traumas. Research in another area of developmental psy- 
chopathology, that of conduct disorder, recently showed that only the combined presence of specific genetic (low expression of monoamine oxidase A) and environmental conditions (child maltreatment) increased the risk for developing antisocial behavior, while each condition in isolation had no effect (Caspi et al., 2002). In the case of PTSD, the implications of the heterogeneity of stressors and of individual and contextual variables for the development of the disorder are still not well understood. We are therefore currently limited in our ability to identify with precision which traumatized children are most at risk for PTSD and should receive selected preventive interventions.

Adult studies indicate that most people exposed to trauma recover with time. Exposure to certain types of trauma seem to involve a greater risk for PTSD. Unanticipated, uncontrollable, life threatening acts of violence, especially if deliberate and committed by trusted adults, have been found to carry worse consequences for the victims than other types of traumas such as accidents or natural calamities. In general, the more direct the exposure to a traumatic event, and the greater the perceived threat to one's own person, the higher the risk for developing PTSD.

Little evidence exists demonstrating that specific symptoms or features of the acute trauma-induced distress predict later PTSD. For instance, the intensity of the acute autonomic reaction has been found to predict PTSD in some studies, but others have found the opposite. Recently, a new sceening instrument, the Screening Tool for Early Predictors of PTSD (STEPP), has been piloted in a pediatric truma center (Winston et al., 2003). The STEPP takes only a minutes to complete and seems to have the high sensitivity required of a screening instrument.

Given the current limitations in identifying with precision traumatized children more likely to develop posttraumatic psychopathology, a reasonable approach to prevention would be to develop relatively brief and inexpensive interventions that can be offered to all children immediately after a severe trauma, with special attention to those at increased risk because of preexisting depression or anxiety, previous history of trauma, lack of family support, and family history of PTSD. However, the data on single-session early psychological debriefing in adults do not support its efficacy, and even suggest a iatrogenic effect with increased odds of developing PTSD symptoms (Bisson, 2003). Multiple-session preventive interventions (usually 4-5 sessions) have received limited testing in adults, with encouraging results, but have not been studied in children. Pharmacological approaches to prevention of PTSD have also been proposed. Specifically, the administration of propranolol to reduce post-traumatic autonomic activation has been pilot-tested in a small sample of adults (Pittman et al., 2002).

It still remains to be determined if one type of preventive intervention can be applied across various types of trauma, or if different trauma-specific interventions need to be developed. Overall, the task of preventing PTSD appears more challenging in children than in adults, because, in addition to trauma and victim characteristics, factors such as stage of development and family variables need to be taken into account in developing interventions.

\section{TREATMENT OF POST-TRAUMATIC PSYCHOPATHOLOGY}

Both cognitive-behavioral psychotherapy (CBT) and selective serotonin reuptake inhibitor medication (SSRI) have been found to be of benefit in adults with PTSD. Few randomized controlled clinical trials have been conducted in children with PTSD (for a review, see Pine \& Cohen, 2002). At least 6 controlled trials of CBT have been completed in sexually abused children. CBT was reported to be better than usual community treatment or non-specific supportive therapy in decreasing PTSD and depression symptoms and improving social competence, with a small to medium effect size (Deblinger et al., 1999; Cohen \& Mannarino, 1997). One randomized controlled trial of CBT in middle school children who had been exposed to violence and had PTSD symptoms has been recently reported (Stein et al., 2003). CBT was administed to groups of children in school according to a specifically prepared manual. Compared with children on the wait-list (i.e., receiving no treatment), children given CBT were found to have a statistically significant decline in scores of PTSD symptoms, depression, and psychosocial dysfunction.

The application of CBT techniques to the treatment of PTSD is well theoretically grounded. Traumas trigger reactions at both the cortical and subcortical level of the brain. The activation of the fear circuitries lead to multiple changes, including autonomic arousal, activation of the hypothalamic-pituitary-adrenal axis, emotional numbing, feelings of derealization, conditioning of peritraumatic stimuli, and avoidance. In parallel, conscious awareness of the trauma undermines the safety assumption of the child, leading to cognitive distortions in interpretating experience and anticipating future events. CBT aims to address all of these core components of post-trau- 
matic psychopathology. Manualized CBT typically includes relaxation training, cognitive restructuring, exposure (either imaginary or in vivo according to the circumstances), social-problem solving, behavioral homework assignments and psychoeducation of the child and family).

A CBT course usually consists of weekly hourly sessions for about 12 weeks. CBT has been utilized for individual and group interventions. Because the parental response to trauma has a strong influence on their children's reactions (e.g., risk for PTSD is greater for those children whose parents are more anxious and depressed), it is important to include the parent in the child treatment. Research is in progress to clarify which specific ingredients of CBT are most therapeutically active and needed for effective treatment.

In particular, attention has been focused on the role of exposure and the relative therapeutic value of the cognitive vs. behavioral components. Exposure has been found to be critical for the efficacy of CBT in adults suffering from PTSD, obsessive-compulsive disorder, agoraphobia, and phobias in general. The role of exposure in CBT for children is therefore suspected to be equally important. In PTSD, exposure usually means evocation of trauma-related memories and emotions (imaginary exposure) in a therapeutic environment with the purpose of desensitizing the child from the their anxiogenic effects. In other cases, it involves in vivo exposure to places or situations that had become conditioned by the trauma and cause agoraphobia and avoidance. The use of exposure techniques can raise concerns about possible re-traumatization and other iatrogenic effects. Gradual exposure techniques have been developed and applied to children in an effort to increase the level of control on anxiety and minimize risks.

No controlled trials of SSRI medication in children with PTSD have been reported yet. The efficacy of these agents in adult PTSD and in children with other types of anxiety disorders, in addition to depression, provides a clear rationale for such trials.

Research involving children with PTSD presents special challenges. Traumas are usually unanticipated, and researchers have to propose research participation to children and families that are enduring considerable stress and may not have the concentration and motivation to consider research. The impact of development and family variables on designing and implementing interventions add other degrees of complexity.

\section{CONCLUSIONS}

Prevention and treatment of post-traumatic psychopathology among children have become important items of the mental health agenda. Though little research has been conducted so far, there is growing interest among researchers. For prevention, a better understanding of risk and protective factors for PTSD following a trauma is needed in order to develop targeted interventions for high risk children. For treatment, identification of the critical ingredients of CBT may lead to more precise, effective, and affordable interventions. Pharmacological treatments are worth pursuing for selected patients. The combined application of pharmacological and psychotherapeutic approaches should be studied. More in general, what is especially promising for the treatment of PTSD is the presence of theoretically driven models that are grounded on the recent understanding of the brain circuitries of fear and anxiety. How to deal with the psychological consequences of indirect exposure to traumatic events, most typically terrorism, has become a timely and crucial area of inquiry with enormous implications. In fact, to successfully address this challenge will mean not only to improve the health and well being of traumatized individuals, but to avert the main aim and purpose of terrorism.

\section{REFERENCES}

American Psychiatric Association (1994). Diagnostic and Statistical Manual of Mental Disorders (DSM-IV), 4th edition. American Psychiatric Press: Washington, DC.

Breslau N. (2002). Epidemiologic studies of trauma, posttraumatic stress disorder, and other psychiatric disorders. Canadian Journal of Psychiatry 47, 923-929.

Bisson J.I. (2003). Single-session early psychological interventions following traumatic events. Clinical Psychology Review 23, 481-499.

Caspi A., McClay J., Moffitt T.E., Mill J., Martin J., Craig I.W., Taylor A. \& Poulton R. (2002). Role of genotype in the cycle of violence in maltreated children. Science 297, 851-854.

Cohen J.A. \& Mannarino A.P. (1997). A treatment study for sexually abused preschool children. Journal of the American Academy of Child and Adolescent Psychiatry 36, 1228-1235.

Costello E.J., Erkanli A., Fairbank J.A. \& Angold A. (2002). The prevalence of potentially traumatic events in childhood and adolescence. Journal of Trauma and Stress 15, 99-112.

Deblinger E., Steer R.A. \& Lippman J. (1999). Two-year follow-up study of cognitive behavioral therapy for sexually abused children suffering post-traumatic stress symptoms. Child Abuse and Neglect $23,1371-1378$.

Friedman M.J., Foa E.B. \& Charney D.S. (2003). Toward evidencebased early interventions for acutely traumatized adults and children. Biological Psychiatry 53, 765-768.

Heim C., Newport D.J., Heit S., Graham,Y.P., Wilcox M., Bonsall R., Miller A.H. \& Nemeroff C.B. (2000). Pituitary-adrenal and autonomic responses to stress in women after sexual and physical abuse in 
childhood. Journal of the American Medical Association 284, $92-$ 597.

Pine D.S. \& Cohen J.A. (2002). Trauma in children and adolescents: risk and treatment of psychiatric sequelae. Biological Psychiatry 51, 519-531.

Pittman R.K., Sanders K.M., Zusman R.M., Healy A.R., Lasko N.B., Cahill L. \& Orr S.P. (2002). Pilot study of secondary prevention of posttraumatic stress disorder with propranolol. Biological Psychiatry 51, 189-1992.

Stein B.D., Jaycox L.H., Kataoka S.H., Wong M., Tu W., Elliott M.N.
\& Fink A. (2003). A mental health intervention for schoolchildren exposed to violence. Journal of the American Medical Association 290, 603-611.

Stevenson J. (1999). The treatment of the long-term sequelae of child abuse. Journal of Child Psychology and Psychiatry 40, 89-111.

Winston F.K., Kassam-Adams N., Gracia-Espana F., Ittenbach R. \& Cnaan, A. (2003). Screening for risk of persistent posttraumatic stress in injured children and their parents. Journal of the American Medical Association 290, 603-611. 